\title{
Nuclear Polarization Corrections for the S-Levels of Electronic and Muonic Deuterium
}

\author{
Yang Lu and R. Rosenfelder \\ Paul Scherrer Institute, CH-5232 Villigen PSI, Switzerland.
}

\begin{abstract}
We calculate the second-order corrections to the atomic energy level shifts in ordinary and muonic deuterium due to virtual excitations of the deuteron which are important for ongoing and planned precise experiments in these systems. For light atoms a method can be used in which the shift is expressed as integrals over the longitudinal and transverse inelastic structure functions of the nucleus. We employ the structure functions arising from separable NN potentials of the Yamaguchi and Tabakin form which can be evaluated analytically. Special emphasis is put on gauge invariance which requires a consistent inclusion of interaction currents and seagull terms. The effect of the D-wave component of the deuteron is investigated for the leading longitudinal contribution. We also estimate the shift for pionic deuterium.
\end{abstract}


1. Recently the isotope shift of the $2 S-1 S$ transition in electronic hydrogen and deuterium has been measured with a thirty-fold increase in accuracy compared to previous experiments [1] and prospects are good to increase the present accuracy of $37 \mathrm{ppb}$ (or $22 \mathrm{kHz}$ ) by another order of magnitude. At this level not only various QED corrections and the finite size of the deuteron are important but also virtual excitations of the deuteron (the so-called nuclear polarization) cannot be neglected anymore. Using a rough square-well model of the deuteron and the closure approximation the 1S shift due to virtual Coulomb excitation was estimated to be about $-19 \mathrm{kHz}$ [2] which is just the present experimental uncertainty. In light and heavy muonic atoms the nuclear polarization shift generally limits the accuracy with which nuclear sizes can be extracted since a reliable calculation of these corrections requires knowledge of the whole nuclear spectrum [3]. The deuteron is unique among all nuclei in that this information is available: the quantum-mechanical 2-body problem is solvable and realistic NN potentials describe bound and scattering states rather well. Therefore, unlike the usual case, nuclear polarization corrections are calculable for the deuteron - presumably with an accuracy at the percent level.

It is the purpose of the present note to evaluate this shift within a more realistic model for the deuteron, avoiding the use of the closure approximation and including also transverse excitations which have been neglected up to now. For the purpose of a planned experiment at PSI with muonic hydrogen [4] which may be extended to deuterium [5] we also evaluate the corresponding shifts in the muonic case. It should be noted that this does not amount to a simple rescaling of the electronic results: since nuclear excitations are of the order of a few $\mathrm{MeV}$ the electron is higly relativistic in the virtual state whereas the muon can be treated nonrelativistically to a good approximation. This leads to different weighting of the individual excitations and therefore to quite different results.

2. We will calculate the nuclear polarization shifts in the atomic S-states of the lepton. These are more difficult to evaluate than the corresponding shifts in higher orbits where only the longest range multipole is of importance and therefore the only nuclear structure information needed is the electric dipole polarizability of the nucleus. In contrast, many multipoles contribute to virtual excitations from atomic S-states since there is an overlap between leptonic and nuclear wavefunctions. In light nuclei, however, the relevant scales (Bohr radius vs. nuclear radius) are vastly different so that the S-wave lepton wavefunction can be considered as approximately constant over the nuclear volume $\phi_{n 0}(\mathbf{x}) \simeq \phi_{\mathrm{av}}$ : the lepton then just acts as a static source with four-momentum $k=(m, \mathbf{0})$.

We will evaluate the S-wave nuclear polarization shifts along the lines of ref. [6], i.e. not by a multipole decomposition but by integrating over the inelastic structure functions of the nucleus. The diagrams which contribute to nuclear polarization are shown in Fig. 1. Note that we need the "seagull" contribution of Fig. 1 (c) for gauge invariance in a nonrelativistic sytem 
like the nucleus. Under the mentioned simplifications one obtains

$$
\Delta \epsilon_{n 0}=-\frac{(4 \pi \alpha)^{2}}{m}\left|\phi_{\mathrm{av}}\right|^{2} \operatorname{Im} \int \frac{d^{4} q}{(2 \pi)^{4}} t_{\mu \nu}(q, k) D^{\mu \rho}(q) D^{\nu \tau}(-q) \bar{T}_{\rho \tau}(q,-q)
$$

where

$$
t_{\mu \nu}(q, k)=\frac{k \cdot q g_{\mu \nu}+(k-q)_{\mu} k_{\nu}+k_{\mu}(k-q)_{\nu}}{(k-q)^{2}-m^{2}+i \epsilon}
$$

is the leptonic tensor, $\alpha=e^{2}=1 / 137.036$ the fine-structure constant and $m$ is the lepton mass. Furthermore, $D^{\mu \rho}(q)$ denotes the photon propagator and $\bar{T}_{\rho \tau}(q,-q)$ the forward virtual nuclear Compton amplitude. To be more precise, the latter is that part of the full Compton amplitude in which the nucleus is in an excited intermediate state. It can be expressed in terms of its imaginary part, i.e. by the inelastic longitudinal and transverse structure functions

$$
S_{L / T}=\sum_{N \neq 0} \delta\left(\omega^{\prime}+E_{0}-E_{N}\right)\left|<\psi_{N}\right| \mathcal{O}_{L / T}\left|\psi_{0}>\right|^{2} .
$$

Here $\omega^{\prime}=\omega-q^{2} / 4 M$ is the internal excitation energy, $E_{0}<0$ the ground state energy and $\mathcal{O}_{L / T}$ are the operators for longitudinal and transverse excitations repectively. In Coulomb gauge one obtains [6]

$$
\begin{aligned}
\Delta \epsilon_{n 0}=-8 \alpha^{2} \bar{R}_{n 0}\left|\phi_{n 0}(0)\right|^{2} \int_{0}^{\infty} d q & \left\{\int_{0}^{\infty} d \omega\left[K_{L}(q, \omega) S_{L}(q, \omega)+K_{T}(q, \omega) S_{T}(q, \omega)\right]\right. \\
& \left.+R_{S}(q)\right\} f^{2}(q) .
\end{aligned}
$$

Here $\bar{R}_{n 0}$ is a correction factor for the variation of the leptonic wave function over the nucleus, $q$ is the magnitude of the three-momentum transfer and $\omega$ the energy transfer to the nucleus. The kernels $K_{L, T}(q, \omega)$ are given in the appendix of ref. [6] for fully relativistic kinematics of the lepton. $R_{S}(q)$ is the contribution from the internal seagull Ð. Finally, $f(q)=(1+$ $\left.q^{2} /\left(0.71 \mathrm{GeV}^{2}\right)\right)^{-2}$ describes the electromagnetic formfactor of the nucleon. Actually eq. (4) not only holds in the Coulomb gauge but is gauge invariant provided $q^{\mu} \bar{T}_{\mu \nu}(q,-q)=0$. This in turn requires current conservation and special conditions for the seagull term 8, 9] which will be discussed below.

3. Unlike ref. [6] where a phenomenological model for the structure functions of ${ }^{12} \mathrm{C}$ had to be used, the deuteron allows for a consistent calculation of these quantities after a nucleon-nucleon interaction has been chosen. For simplicity we take a separable potential of the form $(M$ is the nucleon mass)

$$
V\left(\mathbf{p}, \mathbf{p}^{\prime}\right)=-\frac{\lambda}{M} g(p) g\left(p^{\prime}\right) .
$$

\footnotetext{
${ }^{1}$ The center-of-mass seagull term is part of the two-photon recoil correction [7]. Note also that the part of the Compton amplitude in which the nucleus remains in its ground state has already been included by solving the bound state problem for the lepton with a static potential.
} 
This is not realistic in the modern sense (it lacks the one-pion exchange tail and all other complications of the NN force) but it gives a fairly good description of the low-energy NN interaction which should be sufficient for the present accuracy of isotope shift experiments. Most important for the present purposes it allows for an analytic evaluation of the structure functions. For example, the longitudinal structure function is obtained as [10]

$$
S_{L}(q, \omega)=\int d^{3} p\left|\psi_{0}(\mathbf{p}-\mathbf{q} / 2)\right|^{2} \delta\left(\omega^{\prime}+E_{0}-\frac{p^{2}}{M}\right)+\frac{\lambda M}{\pi} \operatorname{Im}\left(\frac{I^{2}\left(\omega^{\prime}+E_{0}, q\right)}{1+\lambda C\left(\omega^{\prime}+E_{0}\right)}\right) .
$$

Here the functions $C(E)$ and $I(E, q)$ are given by

$$
\begin{aligned}
C(E) & =\int d^{3} p \frac{g^{2}(p)}{M E-p^{2}+i \epsilon} \\
I(E, q) & =\int d^{3} p \psi_{0}(\mathbf{p}-\mathbf{q} / 2) \frac{g(p)}{M E-p^{2}+i \epsilon} .
\end{aligned}
$$

Note that the internal charge operator is

$$
\hat{\rho}(\mathbf{q})=\exp \left(\frac{i}{2} \mathbf{q} \cdot \hat{\mathbf{r}}\right)
$$

where the factor $1 / 2$ arises from the transformation to internal coordinates (a "hat" indicates an operator in the nuclear Hilbert space). The ground state wave function is simply given by

$$
\psi_{0}(\mathbf{p})=N \frac{g(p)}{p^{2}-M E_{0}} .
$$

Also note that the first term in eq. (6) is the impulse approximation to the structure function whereas the last one describes the final-state interaction. Due to the simple form of eq. (5) it only acts in states with angular momentum zero which should be a good approximation for low-energy processes. For the Yamaguchi choice [11]

$$
g_{Y}(p)=\frac{1}{p^{2}+\beta^{2}}
$$

all integrals can be performed analytically. Details will be given elsewhere.

The transverse nuclear polarization shift receives different contributions : first, we have the contribution from the transverse (with respect to $\mathbf{q}$ ) part of the convection current

$$
\hat{\mathbf{J}}^{\text {(conv) }}(\mathbf{q})=\frac{1}{2 M}\left[\hat{\mathbf{p}}, \exp \left(\frac{i}{2} \mathbf{q} \cdot \hat{\mathbf{r}}\right)\right]
$$

which does not have a final-state interaction term since all excited states necessarily have at least angular momentum one. Second, the spin current

$$
\hat{\mathbf{J}}^{(\text {spin) }}(\mathbf{q})=\frac{i}{2 M}\left[\mu_{p}\left(\hat{\boldsymbol{\sigma}}_{p} \times \mathbf{q}\right) \exp \left(\frac{i}{2} \mathbf{q} \cdot \hat{\mathbf{r}}\right)+\mu_{n}\left(\hat{\boldsymbol{\sigma}}_{n} \times \mathbf{q}\right) \exp \left(-\frac{i}{2} \mathbf{q} \cdot \hat{\mathbf{r}}\right)\right]
$$


involves the magnetic moments and spins of neutron and proton and gives rise to spin-flip excitations. Since the procedure of calculating the transverse structure with these currents is similar to the longitudinal case the explicit expressions will not be given here. Note that the operators (9), (12) and (13) obey current conservation in the form

$$
<\mathbf{p}^{\prime}\left|[\hat{T}, \hat{\rho}(\mathbf{q})]-\mathbf{q} \cdot\left(\hat{\mathbf{J}}^{(\text {conv })}(\mathbf{q})+\hat{\mathbf{J}}^{(\text {spin })}(\mathbf{q})\right)\right| \mathbf{p}>=0
$$

where $\hat{T}$ is the kinetic energy operator.

4. A separable potential of the type (5) is nonlocal and equivalent to a momentum-dependent potential

$$
\hat{V}=-\frac{\lambda}{M} \int d^{3} r d^{3} x g\left(\mathbf{r}+\frac{1}{2} \mathbf{x}\right) g\left(\mathbf{r}-\frac{1}{2} \mathbf{x}\right) \exp (-i \hat{\mathbf{p}} \cdot \mathbf{x} / 2)|\mathbf{r}><\mathbf{r}| \exp (-i \hat{\mathbf{p}} \cdot \mathbf{x} / 2) .
$$

Minimal coupling $\hat{\mathbf{p}}_{p} \longrightarrow \hat{\mathbf{p}}_{p}-e \mathbf{A}\left(\mathbf{r}_{p}\right)$ in the two-body Hamiltonian $\hat{H}=\hat{T}+\hat{V}$ then produces a power series in the electromagnetic coupling constant $e$

$$
\hat{H}=\left.\hat{H}\right|_{A=0}-e \int d^{3} y \hat{J}_{\mu}(\mathbf{y}) A^{\mu}(\mathbf{y})+\frac{e^{2}}{2} \int d^{3} y d^{3} z A_{i}(\mathbf{y}) A_{j}(\mathbf{z}) \hat{B}_{i j}(\mathbf{y}, \mathbf{z})+\ldots
$$

The linear terms due to the potential generate an interaction current

$$
e \Delta \hat{J}_{k}(\mathbf{y})=-\left.\frac{\delta \hat{V}}{\delta A_{k}(\mathbf{y})}\right|_{A=0}
$$

whereas the second-order terms give rise to the interaction seagull

$$
e^{2} \Delta \hat{B}_{i j}(\mathbf{y}, \mathbf{z})=\left.\frac{\delta^{2} \hat{V}}{\delta A_{i}(\mathbf{y}) \delta A_{j}(\mathbf{z})}\right|_{A=0} .
$$

With the separable potential (15) one obtains the following expression for the matrix element of the interaction current

$$
<\mathbf{p}^{\prime}|\Delta \hat{\mathbf{J}}(\mathbf{q})| \mathbf{p}>=-\frac{\lambda}{2 M}\left(\nabla_{p}+\nabla_{p^{\prime}}\right) \int_{0}^{1} d u g\left(\mathbf{p}-u \frac{\mathbf{q}}{2}\right) g\left(\mathbf{p}^{\prime}+(1-u) \frac{\mathbf{q}}{2}\right)
$$

which satisfies

$$
<\mathbf{p}^{\prime}|[\hat{V}, \hat{\rho}(\mathbf{q})]-\mathbf{q} \cdot \Delta \hat{J}(\mathbf{q})| \mathbf{p}>=0
$$

so that all together our currents are conserved under the time-evolution of the full Hamiltonian. Similarly one obtains the following matrix element of the interaction seagull

$$
\begin{aligned}
& <\mathbf{p}^{\prime}\left|\Delta \hat{B}_{i j}(\mathbf{q})\right| \mathbf{p}>\equiv<\mathbf{P}_{f}=\mathbf{0}, \mathbf{p}^{\prime}\left|\int d^{3} x \exp (i \mathbf{q} \cdot \mathbf{x}) \Delta \hat{B}_{i j}(\mathbf{x}, \mathbf{0})\right| \mathbf{p}, \mathbf{P}_{i}=\mathbf{0}> \\
& =-\frac{\lambda}{4 M}\left(\nabla_{p}+\nabla_{p^{\prime}}\right)_{i}\left(\nabla_{p}+\nabla_{p^{\prime}}\right)_{j} \int_{-1}^{+1} d u(1-|u|) g\left(\mathbf{p}-u \frac{\mathbf{q}}{2}\right) g\left(\mathbf{p}^{\prime}-u \frac{\mathbf{q}}{2}\right)
\end{aligned}
$$


which comes in addition to the kinematical (internal) seagull $\delta_{i j} / 2 M$.

It is well known [8, 9] that for full gauge invariance of the Compton amplitude a relation between the interaction current and the interaction seagull is needed. In the present case where no overall momentum is transferred to the target it reads

$$
<\mathbf{p}^{\prime}\left|\left[\hat{\rho}^{\dagger}(\mathbf{q}), \Delta \hat{J}_{k}(\mathbf{q})\right]\right| \mathbf{p}>=q<\mathbf{p}^{\prime}\left|\Delta \hat{B}_{3 k}(\mathbf{q})\right| \mathbf{p}>
$$

With the interaction current and interaction seagull as given in eqs. (18) and (21) this relation can be shown to be fulfilled. Thus the nuclear polarization shift is gauge invariant if the interaction terms in current and seagull are taken into account consistently. The seagull contribution in eq. (4) is now explicitly given by

$$
R_{S}(q)=\frac{1+\kappa(q)}{8 m M}\left(\frac{1}{q}-\frac{1}{\sqrt{m^{2}+q^{2}}}\right)
$$

where

$$
\begin{aligned}
& \kappa(q)=\frac{4 \lambda}{q^{2}} \int_{0}^{1} d u \frac{1}{u^{2}}\left[h^{2}(0)-h^{2}(u q)\right] \\
& h(x)=\int d^{3} p \psi_{0}(p) g\left(\mathbf{p}-\frac{\mathbf{x}}{2}\right) .
\end{aligned}
$$

5. We now turn to the numerical results of our calculation. We have evaluated the nuclear polarization shift consistently with the Yamaguchi separable form (5) using the value $\beta=286$ $\mathrm{MeV}$. We have checked numerically that our structure function fulfills the non-energy-weighted sum rule

$$
\int_{0}^{\infty} d \omega S_{L}(q, \omega)=1-F_{0}^{2}(q)
$$

to better than 1 part in $10^{5}$. Here $F_{0}(q)$ is the elastic formfactor calculated directly from the ground state wave function (10). The electric dipole polarizability (which is not the only relevant quantity for S-wave shifts) was found to be $0.613 \mathrm{fm}^{3}$ compared to experimental values of $0.61-0.70 \mathrm{fm}^{3}$ [12. The point rms-radius in this model is $1.92 \mathrm{fm}$ compared to the experimental value $1.96 \mathrm{fm}$. This shows that the simple Yamaguchi parametrization describes the deuteron properties and therefore the low-energy triplet NN-interaction reasonably well. For the spin-flip excitations one also needs the parameters in the singlet channel. Again following Yamaguchi we assume $\beta_{s}=\beta_{t}$ and determine the corresponding strength parameter from the singlet scattering length $a_{s}=-23.69 \mathrm{fm}$.

For non-relativistic point hydrogen wave functions one has

$$
\left|\phi_{n 0}(0)\right|^{2}=\frac{1}{\pi a_{B}^{3}} \frac{1}{n^{3}}
$$


where $a_{B}=1 /\left(\alpha m_{\text {red }}\right)$ is the Bohr radius and $m_{\text {red }}$ the reduced mass of the lepton. Writing

$$
\Delta \epsilon_{n 0}=\frac{\bar{R}_{n 0}}{n^{3}} \Delta \bar{\epsilon}
$$

the shift $\Delta \bar{\epsilon}$ is then independent of the atomic state. We have evaluated the double integral in eq. (4) by Gauss-Legendre numerical integration with up to $3 \times 72$ points. Our results for the different contributions and for the total $\Delta \bar{\epsilon}$ are listed in Table 1. It should be emphasized that the integrand from the transverse convection current has a $1 / q$ - divergence for small $q$ which , however, is exactly cancelled by the seagull due to the gauge condition for the two-photon operator. This can also be seen from eqs. (4) and (23) : at low $q$ the kernel $K_{T}(q, \omega)$ behaves like to $-1 / 4 m q \omega$ [6] and Siegert's theorem tells us that

$$
\int_{0}^{\infty} d \omega \frac{1}{\omega} S_{T}(0, \omega)=\frac{1+\kappa}{2 M}
$$

where $\kappa \equiv \kappa(0)$ is the dipole enhancement factor $(\kappa=0.176$ for the Yamaguchi potential). However, the resulting contribution to the energy shift is exactly opposite in sign to the $q \rightarrow$ 0 -limit of the seagull contribution (23). We have checked numerically that our transverse structure function fulfills the sum rule (29) to sufficient accuracy. Note that in ref. [6] the seagull was represented by eq. (29) for all q; we have found numerically that the extra $q$ dependence only gives a tiny contribution to the full shift.

In Table 1 we therefore give only the combined contribution of transverse convection and seagull excitations. It is seen that it is bigger in electronic deuterium than in muonic deuterium because the electron velocity is higher in the first case. As the spin current contribution vanishes for $q=0$ (see eq. (13)) it can be given separately. However, numerically it turns out to be of no great importance. The same can be said of the interaction terms which nearly cancel the spin contribution. The smallness of the interaction terms is welcome since the nonlocality of the Yamaguchi separable potential is somehow artificial and only partly simulates exchange current effects. It should be kept in mind that the individual contributions are gauge-dependent and that only the total $\Delta \bar{\epsilon}$ is a meaningful physical quantity. The size of the transverse and seagull terms, however, indicates the errors one usually makes if only the longitudinal excitations are taken into account. As to the numerical accuracy, we have checked that the results in Table 1 are accurate to one part in the last digit.

In order to estimate the model dependence of these results we also have calculated the dominating longitudinal contribution for the Tabakin separable potential [13] which describes both attraction at low energies and repulsion at higher energies in the S-wave phase shift. The principal value integrals in eqs. (7) and (8) have now been performed numerically which considerably increased the computing time. Again the sum rule was checked and an electric dipole polarizability of $0.623 \mathrm{fm}^{3}$ was obtained. The values for the shift given in Table 2 are estimated to have an accuracy of about three parts in the last digit. Despite the different 
functional parametrization of $g(p)$ the result for $\Delta \bar{\epsilon}$ is very close to the Yamaguchi one which shows that only low-energy properties of the NN-interaction are important for the nuclear polarization shift.

Finally we also have investigated the influence of the D-state admixture in the deuteron by using the Yamaguchi separable potential with tensor force [14]

$$
g(\mathbf{p})=g_{Y}(p)+\frac{1}{\sqrt{8}} T_{Y}(p) S_{p n}(\mathbf{p})
$$

with

$$
\begin{aligned}
T_{Y}(p) & =-\frac{t p^{2}}{\left(p^{2}+\gamma^{2}\right)^{2}} \\
S_{p n}(\mathbf{p}) & =3 \frac{\boldsymbol{\sigma}_{p} \cdot \mathbf{p} \boldsymbol{\sigma}_{n} \cdot \mathbf{p}}{p^{2}}-\boldsymbol{\sigma}_{p} \cdot \boldsymbol{\sigma}_{n} .
\end{aligned}
$$

The original parameter values of ref. [14 lead to an asymptotic D/S ratio of 0.0285 which is quite reasonable when compared with modern values [15]. The dipole polarizability is calculated to be $0.625 \mathrm{fm}^{3}$. Since the algebra including the tensor force is more involved the sum rule check (to one part in $10^{3}$ ) is nontrivial.

To convert these numbers to the actual nuclear polarization shifts for the S-levels we need the finite size correction factors $\bar{R}_{n 0}$. Using the approximate atomic wavefunctions of ref. 16] one obtains in first order in the ratio nuclear radius/Bohr radius

$$
\bar{R}_{n 0} \simeq 1-3.06 \frac{<r^{2}>^{1 / 2}}{a_{B}} .
$$

The numerical factor in this equation was determined by evaluating the ratio of various moments of the charge distribution with the Yamaguchi ground state wavefunction. Eq. (33) gives $n$-independent correction factors $\bar{R}^{(\mu)}=0.9793$ and $\bar{R}^{(e)}=0.99989$. Of course, on the present level of accuracy one can practically neglect these correction factors.

We estimate the accuracy of our theoretical predictions in the following (rather conservative) way: the accuracy of the calculated longitudinal shift is taken as three times the modeldependence shown in Table 2 and we assign a $20 \%$ error to the transverse current contribution and $50 \%$ one to the interaction pieces. Adding these errors linearly we therefore arrive at the final result for the nuclear polarization shifts in electronic and muonic deuterium

$$
\begin{aligned}
\Delta \epsilon_{n 0}^{(e)} & =(-20.5 \pm 1.3) \frac{1}{n^{3}} \mathrm{kHz} \\
\Delta \epsilon_{n 0}^{(\mu)} & =(-11.6 \pm 0.5) \frac{1}{n^{3}} \mathrm{meV}
\end{aligned}
$$

We also have estimated the nuclear polarization shift in pionic deuterium by replacing the muon mass by the pion mass. The longitudinal and the transverse convection current contribution of 
the present formalism should give a reasonable value also for a heavy spin zero particle because to a good approximation it can be treated nonrelativistically with no difference between a Dirac and a Klein - Gordon description. In this way we obtain

$$
\Delta \epsilon_{n 0}^{(\pi)} \simeq-28 \frac{1}{n^{3}} \mathrm{meV}
$$

For $n=1$ this is a factor of two smaller than the precision aimed at in an ongoing experiment at PSI to measure the strong interaction shifts in pionic hydrogen [17].

If the future isotope shift experiments in electronic deuterium actually reduce the experimental accuracy to about $1 \mathrm{kHz}$ [1] it would be worthwhile to repeat the present calculation with a modern NN-potential like the Paris potential [18. Also it may be useful to employ a general gauge like the " $\alpha$-Lorentz gauge" [19] which interpolates between Coulomb and Lorentz gauge thereby demonstrating explicitly the independence of the numerical results from the gauge parameter $\alpha$. Finally, before discrepancies between theory and experiment in the isotope shifts are taken serious one should include second-order effects also in the analysis of electron-deuteron scattering experiments which extract the root-mean square radius of the deuteron. In the case of ${ }^{12} \mathrm{C}$ similar discrepancies between electron scattering data and muonic

energy shifts seem to disappear [20] when second-order effects are taken into account in the analysis of both experiments.

\section{Acknowledgements}

We thank Andreas Schreiber for helpful discussions and a critical reading of the manuscript. We are grateful to Leo Simons and Pieter Goudsmit for providing us with experimental information. 


\section{References}

[1] F. Schmidt-Kaler, D. Leibfried, M. Weitz and T. W. Hänsch, Phys. Rev. Lett. 70 (1993) 2261

[2] K. Pachucki, D. Leibfried and T. W. Hänsch, Phys. Rev. A 48 (1993) R1

[3] R. Rosenfelder, in: "Muonic Atoms and Molecules", eds. L. A. Schaller and C. Petitjean, Birkäuser (1993), p. 95

[4] E. Zavattini et al., PSI proposal R-93-06.1 (1993)

[5] L. Simons, private communication

[6] R. Rosenfelder, Nucl. Phys. A 393 (1983) 301

[7] H. Grotch and D. R. Yennie, Rev. Mod. Phys. 41 (1969) 350

[8] J. L. Friar and M. Rosen, Ann. Phys. 87 (1974) 289

[9] H. Arenhövel, Z. Phys. A 297 (1980) 129

[10] R. Rosenfelder, Ann. Phys. 128 (1980) 188

[11] Y. Yamaguchi, Phys. Rev. 95 (1954) 1626

[12] J. L. Friar and S. Fallieros, Phys. Rev. C 29 (1984) 232

[13] F. Tabakin, Phys. Rev. 174 (1968) 1208

[14] Y. Yamaguchi and Y. Yamaguchi, Phys. Rev. 95 (1954) 1635

[15] T. E. O. Ericson and M. Rosa-Clot, Ann. Rev. Nucl. Part. Sci. 35 (1985) 271

[16] J. L. Friar, Z. Phys. A 292 (1979) 1

[17] P. F. A. Goudsmit, private communication

[18] M. Lacombe et al., Phys. Rev. C21 (1980) 861

[19] C. Baxter, Ann. Phys. 206 (1991) 221

[20] E. A. J. M. Offerman et al., Phys. Rev. C 44 (1991) 1096 


\section{Table 1 :}

Contributions to the nuclear polarization shift $\Delta \bar{\epsilon}$ ( see eq. (28) ) for electronic (e) and muonic $(\mu)$ deuterium in Coulomb gauge. The Yamaguchi S-wave separable potential has been used throughout. The different contributions are labeled by L : longitudinal; $\mathrm{T}^{(\text {conv })}+\mathrm{S}$ : tranverse convection current + seagull; $\mathrm{T}^{(\mathrm{spin})}$ : transverse spin current; $\Delta(\mathrm{T}+\mathrm{S})$ : interaction transverse current + interaction seagull.

\begin{tabular}{|l|r|r|}
\hline Contribution & $\mathrm{e}[\mathrm{kHz}]$ & $\mu[\mathrm{meV}]$ \\
\hline $\mathrm{L}$ & -18.31 & -11.77 \\
$\mathrm{~T}^{\text {(conv) }}+\mathrm{S}$ & -2.25 & -0.06 \\
$\mathrm{~T}^{\text {(spin) }}$ & +0.33 & +0.03 \\
$\Delta(\mathrm{T}+\mathrm{S})$ & -0.31 & -0.02 \\
total & -20.54 & -11.82 \\
\hline
\end{tabular}

\section{Table 2 :}

Longitudinal nuclear polarization shift $\Delta \bar{\epsilon}$ for different separable NN-potentials.

\begin{tabular}{|l|c|r|}
\hline NN-Potential & e [kHz] & $\mu[\mathrm{meV}]$ \\
\hline Yamaguchi S-wave [11] & -18.31 & -11.77 \\
Tabakin S-wave [13] & -18.54 & -11.92 \\
Yamaguchi S+D-wave [14] & -18.45 & -11.86 \\
\hline
\end{tabular}

\section{Figure caption}

\section{Fig. 1 :}

Second-order contributions to the nuclear polarization shift: (a) box graph, (b) crossed graph, (c) seagull graph. 
This figure "fig1-1.png" is available in "png" format from: http://arxiv.org/ps/nucl-th/9311025v1 